\title{
Microbiota Fúngica en Quesos Artesanales en Saltillo, Coahuila, México
}

\author{
José Luis Arispe-Vazquez; Abiel Sanchez-Arizpe*; Ma. Elizabeth Galindo-Cepeda \\ Universidad Autónoma Agraria Antonio Narro, Departamento de Parasitología, Calzada Antonio Narro \#1923, \\ Buenavista, Saltillo, Coahuila. C.P. 25315. Tel. 844-4110226. \\ *abielsanchez@hotmail.com
}

Fecha recepción: marzo 3 de 2020 Fecha aceptación: abril 7 de 2020

\section{Resumen}

El queso es un alimento importante en la cocina mexicana, el consumo per cápita en México es de $6 \mathrm{~kg}$; sin embargo, puede transportar numerosos hongos dañinos para las personas, como micotoxigénicos. El objetivo del trabajo fue evaluar la microbiota fúngica presente en los quesos artesanales del mercado abierto en Saltillo, Coahuila, México, se obtuvieron cinco tipos de quesos por semana de diferentes orígenes; uno de Saltillo, dos del general Cepeda (A y B), uno de Mazapil, Zacatecas y uno de Ciudad Fernández, San Luis Potosí. Cada queso se cortó en tres niveles (réplicas), y se tomó una muestra de $1 \mathrm{~cm}$ en el norte, sur, este, oeste y centro del queso, con cuatro réplicas, que se colocaron en placas de Petri con medio de cultivo PDA mantenido a $25{ }^{\circ} \mathrm{C} \pm 2{ }^{\circ} \mathrm{C}$ durante $120 \mathrm{~h}$. Los hongos se identificaron utilizando la técnica de Medina y colaboradores. Geotrichum candidum, Fusarium verticillioides, Rhizopus nigricans, Cladosporium sp. y Penicillium sp. fueron identificados morfológicamente, con una incidencia entre 0 y $100 \%$. Fusarium verticillioides y Penicillium sp. son hongos toxigénicos, y Geotrichum candidum causa infección en los bronquios, pulmones y membranas mucosas, llamada geotricosis, por lo que es importante implementar medidas de calidad para garantizar la producción segura de quesos para el consumo humano.

Palabras clave: Hongo; Alimento, Calidad; Producción. 


\title{
Fungal Microbiota in Artisan Cheeses in Saltillo, Coahuila, Mexico
}

\begin{abstract}
Cheese is an important food in Mexican cooking, the per capita consumption in Mexico is $6 \mathrm{~kg}$; however, it can carry numerous harmful fungi for people, as mycotoxigenics. The objective of the work was to evaluate fungal microbiota present in artisanal cheeses from the open market in Saltillo, Coahuila, Mexico, five types of cheeses were obtained per week from different origins; one from Saltillo, two from General Cepeda (A and B), one from Mazapil, Zacatecas and one from Ciudad Fernandez, San Luis Potosi. Each cheese was cut in three levels (replicates), and a $1 \mathrm{~cm}$ sample was taken on the North, South, East, West, and center of the cheese, with four replicates, which were placed in Petri dishes with PDA culture medium kept at $25{ }^{\circ} \mathrm{C} \pm 2{ }^{\circ} \mathrm{C}$ for $120 \mathrm{~h}$. Fungi were identified using the Medina and collaborators technique. Geotrichum candidum, Fusarium verticillioides, Rhizopus nigricans, Cladosporium sp. and Penicillium $\mathrm{sp}$. were identified morphologically, with an incidence ranging from 0 to $100 \%$. Fusarium verticillioides and Penicillium sp. are toxigenic fungi, and Geotrichum candidum cause infection in the bronchial tubes, lungs and mucous membranes, called geotrichosis, so it is important to implement quality measures to guarantee the safe production of cheeses for human consummation.
\end{abstract}

Keywords: Fungi; Food; Quality; Production.

\section{Microbiota Fúngica em Queijos Artesanais em Saltillo, Coahuila, México}

\begin{abstract}
Resumo
O queijo é um alimento importante na culinária mexicana, o consumo per capita no México é de 6 kg; no entanto, pode transportar inúmeros fungos prejudiciais às pessoas, como micotoxigênicas. O objetivo do trabalho foi avaliar a microbiota fúngica presente em queijos artesanais do mercado aberto em Saltillo, Coahuila, México, foram obtidos cinco tipos de queijos por semana, de diferentes origens; um de Saltillo, dois do General Cepeda (A e B), um de Mazapil, Zacatecas e um de Ciudad Fernández, San Luis Potosí. Cada queijo foi cortado em três níveis (réplicas) e uma amostra de $1 \mathrm{~cm}$ foi coletada no norte, sul, leste, oeste e centro do queijo, com quatro réplicas, que foram colocadas em placas de Petri com o meio de cultura PDA mantido a $25{ }^{\circ} \mathrm{C} \pm 2{ }^{\circ} \mathrm{C}$ por $120 \mathrm{~h}$. Os fungos foram identificados pela técnica de Medina e colaboradores. Geotrichum candidum, Fusarium verticillioides, Rhizopus nigricans, Cladosporium sp. e Penicillium sp. foram identificados morfologicamente, com incidência entre 0 e $100 \%$. Fusarium verticillioides e Penicillium sp. são fungos toxigênicos e o Geotrichum candidum causa infecção nos brônquios, pulmões e membranas mucosas, denominada geotricose, por isso é importante implementar medidas de qualidade para garantir a produção segura de queijos para consumo humano.
\end{abstract}

Palavras-chave: Fungo; Alimento; Qualidade; Produção. 


\section{Introducción}

La producción y consumo de quesos en México tiene su origen en la llegada de los españoles hace más de 500 años [1]. El queso ha sido un componente importante de la dieta humana en el mundo, México tiene un consumo per cápita de $6 \mathrm{~kg}$ [2]. Es un producto rico en proteínas, grasas, minerales y vitaminas, además de contener también aminoácidos biológicamente activos, péptidos y bacterias ácido lácticas que pueden ser probióticas [3].

Las enfermedades de transmisión alimentaria (ETAS) constituyen un problema de salud pública creciente en todo el mundo. Se deben a la ingestión de alimentos contaminados por microorganismos o sustancias químicas [4]. La microbiota del queso se caracteriza por la presencia de una gran variedad de bacterias, levaduras y mohos, que interactúan para desempeñar un papel importante durante la fabricación y la maduración del queso [5]. Se han identificado especies de mohos en la leche cruda: Penicillium camemberti, Cladosporium herbarum, Chrysosporium sulfureum, Rhizomucor fuscus, Rhizomucor plumbeus y Trichothecium domesticum [6]. La presencia de contaminantes fúngicos en alimentos no sólo se interpreta como una fuente potencial de deterioro, sino como la posibilidad de encontrar micotoxinas [7].

Los quesos artesanales poseen una gran variedad de especies de levaduras, principalmente pertenecientes a los géneros Debaryomyces, Geotrichum, Kluyveromyces, Candida, Pichia y Yarrowia [8-11], aunque la prevalencia de varias especies puede estar influenciada por el tipo de queso [12], el tiempo de maduración, la forma en como son elaborados, debido a que la mayoría son artesanales y en muchas ocasiones las condiciones higiénico sanitarias no son las más adecuadas, es decir, son muy deficientes. Otros microorganismos igualmente utilizados en la fabricación de quesos son; Propionibacterium freudenreichii, Brevibacterium liinens, Debaryomyces hansenii, Geotrichum candidum, Penicillium roqueforti y $P$. camemberti [13].

Es importante conocer la actividad metabólica de los microrganismos en el interior del queso y aprovechar este conocimiento para producir nuevos o mejores alimentos fermentados, aumentar el rendimiento y mejorar la calidad, seguridad y composición de los alimentos [14]. El objetivo del trabajo fue evaluar la microbiota fúngica presente en los quesos artesanales del mercado abierto en Saltillo, Coahuila, México.

\section{Materiales y métodos}

\section{Área de estudio}

Las muestras de queso maduro artesanal tipo "ranchero" elaboradas a partir de leche de vaca, se obtuvieron del mercado abierto de Saltillo, Coahuila, México, cuyas coordenadas son $25^{\circ} 24^{\prime} 03.5^{\prime \prime} \mathrm{N} 101^{\circ} 00^{\prime} 35.0^{\prime \prime} \mathrm{W}$.

\section{Muestreo}

El muestreo se realizó del 22 agosto al 10 de octubre de 2019, cada semana se obtenían 5 tipos de quesos del mercado, uno de Saltillo, dos de General Cepeda (A y B) uno de Mazapil, Zacatecas y uno de Ciudad Fernández, San Luis Potosí (Figura 1), es decir, un total de 20 muestras analizadas.
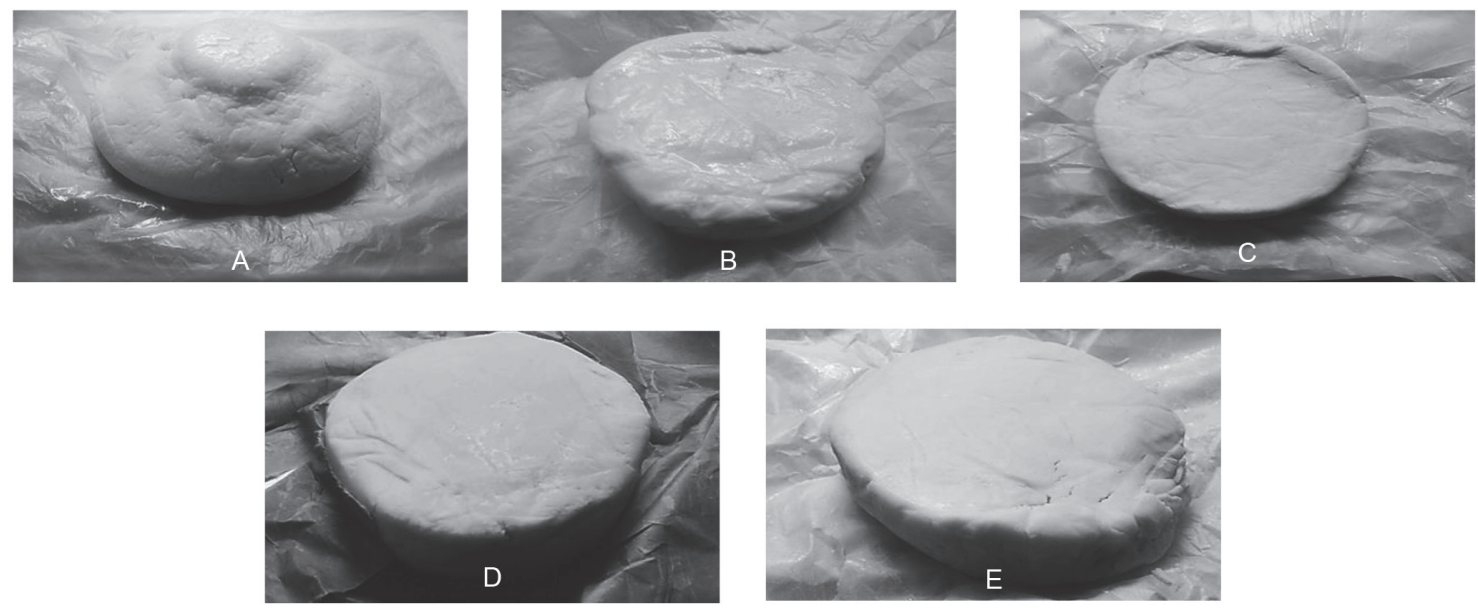

Figura 1. Quesos de distintas regiones; $A=$ Saltillo, $B=$ General Cepeda $(A$ y B $), C=$ Mazapil, Zacatecas y $D=$ Ciudad Fernández, San Luis Potosí. 


\section{Aislamiento}

Los quesos se estudiaron $3 \mathrm{~h}$ después de adquirirlos y se cortaron transversalmente en tres partes iguales. Posteriormente con un sacabocados se obtuvo una muestra de $1 \mathrm{~cm}$ de ancho y $10 \mathrm{~cm}$ de largo de cada cuarta parte del queso, replicado cuatro veces por tipo de queso (Figura 2).

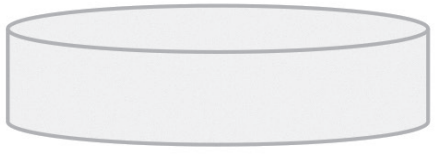

A

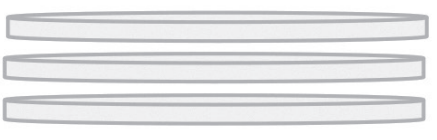

B

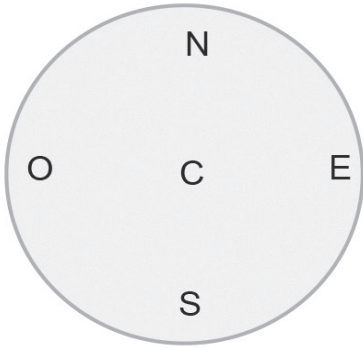

Figura 2. Esquema utilizado para la evaluación de la micobiota en los quesos, $A=$ Queso completo, $B=$ Queso en estratos, $\mathrm{C}=$ Puntos de evaluación.

Solamente se colocaba la mitad de la muestra de queso obtenida $(0.5 \mathrm{~cm})$ sobre una placa de Petri (de 8.5 de diámetro) con medio de cultivo Papa Dextrosa Agar (PDA, Bioxon) + Antibiótico (Gentamicina, $1 \mathrm{~mL} \mathrm{~L}^{-1}$ ), para evitar el crecimiento de bacterias. Posteriormente se rotularon las placas y se sellaron, y por último se incubaron a una temperatura de $25^{\circ} \mathrm{C} \pm 2{ }^{\circ} \mathrm{C}$ durante $120 \mathrm{~h}$.

\section{Purificación de los patógenos mediante cultivos monoconidiales}

De cada colonia de hongo que creció en las placas, se extrajeron muestras de $5 \mathrm{~mm}$ de diámetro y se pusieron en tubos de ensayo con $9 \mathrm{~mL}$ de agua destilada previamente esterilizada. Con ayuda de una micropipeta, se extrajo solo $30 \mu \mathrm{L}$ y se colocó en una placa de Petri con medio de cultivo PDA y con una varilla estéril se dispersó. A las $24 \mathrm{~h}$ se extrajo un solo conidio germinado y éste se colocó en placas de Petri nuevamente con medio PDA, las cuales se incubaron a $25^{\circ} \mathrm{C} \pm 2{ }^{\circ} \mathrm{C}$ por $120 \mathrm{~h}$.

\section{Identificación de los patógenos}

Una vez incubadas las placas, los hongos desarrollados se identificaron mediante criterios morfológicos de Samson y Barnett y Hunter [15-16], utilizando la técnica de cinta adhesiva transparente en azul de lactofenol sobre una porta objetos.

\section{Análisis de resultados}

La incidencia se reportó como porcentaje de hongos presentes en cada punto evaluado por estrato por repetición y tipo de queso.

\section{Resultados y discusión}

\section{Identificación de hongos}

De acuerdo a las características macro y micro morfológicas, se identificaron 2 géneros (Cladosporium y Penicillium) y 3 especies de hongos (Rhizopus nigricans, Geotrichum candidum y Fusarium verticillioides) presentes en los quesos (Figura 3) (Tabla 1).

Tabla 1. Incidencia (\%) de hongos en las distintas localidades

\begin{tabular}{cccccc}
\hline Género & Saltillo & $\begin{array}{c}\text { General } \\
\text { Cepeda (A) }\end{array}$ & $\begin{array}{c}\text { General } \\
\text { Cepeda (B) }\end{array}$ & $\begin{array}{c}\text { San } \\
\text { Luis Potosí }\end{array}$ & Zacatecas \\
\hline Geotrichum candidum & 30,70 & 39,34 & 53,34 & 73,33 & 100 \\
Fusarium verticillioides & 36,03 & 0 & 0 & 0 & 0 \\
Cladosporium sp. & & 20 & 0 & 0 & 0 \\
Penicillium sp. & 26,67 & 40,66 & 33,33 & 26,67 & 0 \\
Rhizopus nigricans & 6,60 & 0 & 13,33 & 0 & 0 \\
\hline Total & 100 & 100 & 100 & 100 & 100 \\
\hline
\end{tabular}




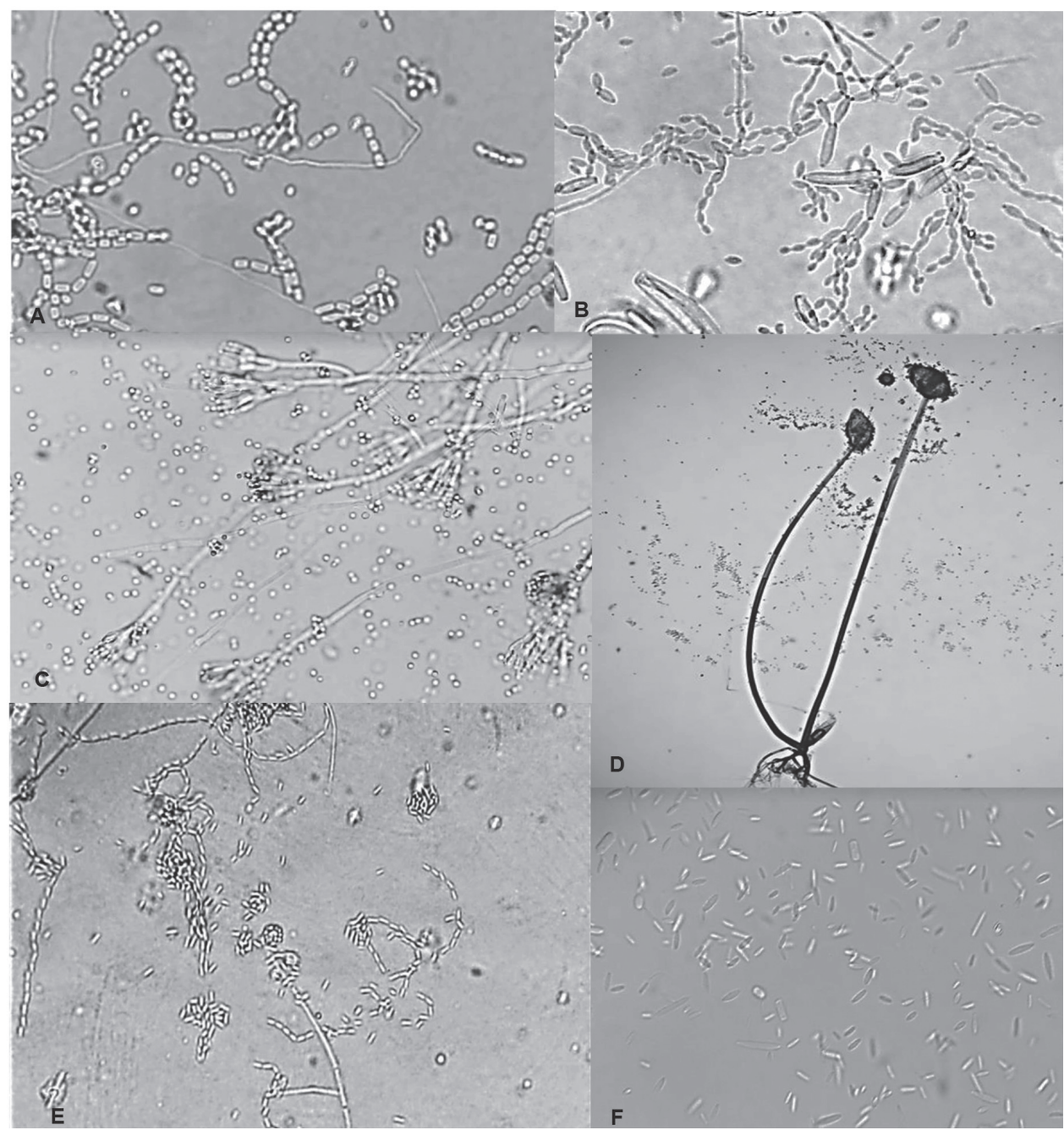

Figura 3. Hongos presentes en quesos; $\mathrm{A}=$ Artrosporas de Geotrichum candidum, $\mathrm{B}=$ Conidios de Cladosporium sp., C= Estructura de Penicillium sp., D= Estructura de Rhizopus nigricans, E= Microconidios en cadena y F= Macroconidos con microconidios de Fusarium verticillioides.

En una investigación previa se estudió la diversidad de los hongos presentes en el queso Paipa (único queso típico madurado de Colombia) [17] y se encontraron especies de los géneros: Penicillium, Aspergillus, Fusarium, Phoma, Cladosporium, Botrytis y Geotrichum. En otro estudio se reportaron hongos presentes en quesos artesanales de cinco zonas de la ciudad de Maracaibo, estado Zulia, Venezuela. En la zona I identificaron a especies de los géneros Penicillium (64,71\%), Aspergillus (21,56\%), Cladosporium $(5,88 \%)$, Drechslera y Curvularia (3,92\%). En la zona II Cladosporium (48,48\%), Aspergillus (24,24\%), Penicillium (15,15\%) y Drechslera (12,12\%). En la zona III Penicillium (78,72\%) y Aspergillus (21,27\%). En la zona IV Aspergillus $(64,31 \%)$ y Penicillium (35,68\%). En la zona $V$
Penicillium (80,00\%), Aureobasidium (14,28\%) y Aspergillus (5,71\%). Hongos similares como contaminantes de alimentos, reportados en este trabajo fueron Penicillium y Cladosporium con una incidencia del 25,47 y $4,0 \%$ respectivamente [18]. Desde el punto de vista higiénico-sanitario los riesgos del consumo de este tipo de quesos implica un alto riesgo de enfermedades, debido a que no se conocen los estándares de calidad durante todo el proceso de su elaboración, en el presente trabajo el hongo con más incidencia en los diferentes quesos estudiados fue Geotrichum candidum (59,34\%) (Figura 4 y 5), una especie dimórfica importante en quesos madurados que afecta a su bioquímica durante el proceso de maduración y en la elaboración de yogures a los que confiere una textura sedosa muy especial [19]. 
Sin embargo Geotrichum candidum es un hongo patógeno oportunista que produce una infección en bronquios y pulmones en animales y el hombre denominada geotricosis, que también afecta a las mucosas (orales y vaginales) [20]. Otra especie identificada en los quesos mexicanos examinados fue Fusarium verticillioides (54,54\%) (una alta incidencia de este patógeno puede representar una producción mayor de metabolitos perjudiciales para las personas). En Argentina se realizó un análisis de riesgo en la elaboración de quesos artesanales encontrándose Escherichia coli en el interior del queso y Fusarium moniliforme (Actualmente Fusarium verticillioides) en la superficie, lo que implica un riesgo para el consumidor cuándo se consumen frescos y la posibilidad de ingerir metabolitos tóxicos [21].

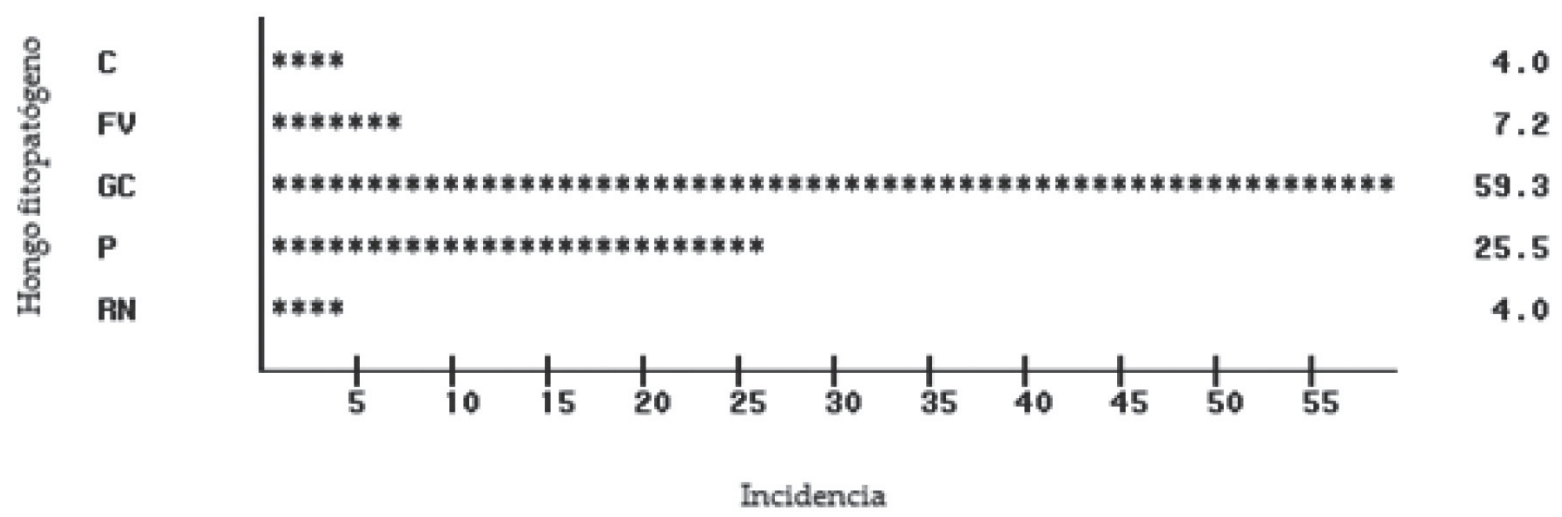

Figura 4. Porcentaje total de los hongos aislados en muestras de quesos artesanales en cinco localidades. $\mathrm{C}=$ Cladosporium sp., $\mathrm{FV}=$ Fusarium verticillioides, $\mathrm{GC}=$ Geotrichum candidum, $\mathrm{P}=$ Penicillium sp., $\mathrm{RN}=$ Rhizopus nigricans.

El número de microorganismos de los quesos en estudio fue mayor en los puntos Este y Centro y menor en los puntos Norte, Sur y Oeste (Figura 5), esto debido a las condiciones higiénicas insuficientes de las personas o personal encargado de su elaboración, así como de los insumos necesarios (calidad de la leche y tipo de cuajo o coagulante), las condiciones ambientales involucrada, la forma de almacenamiento y comercialización.

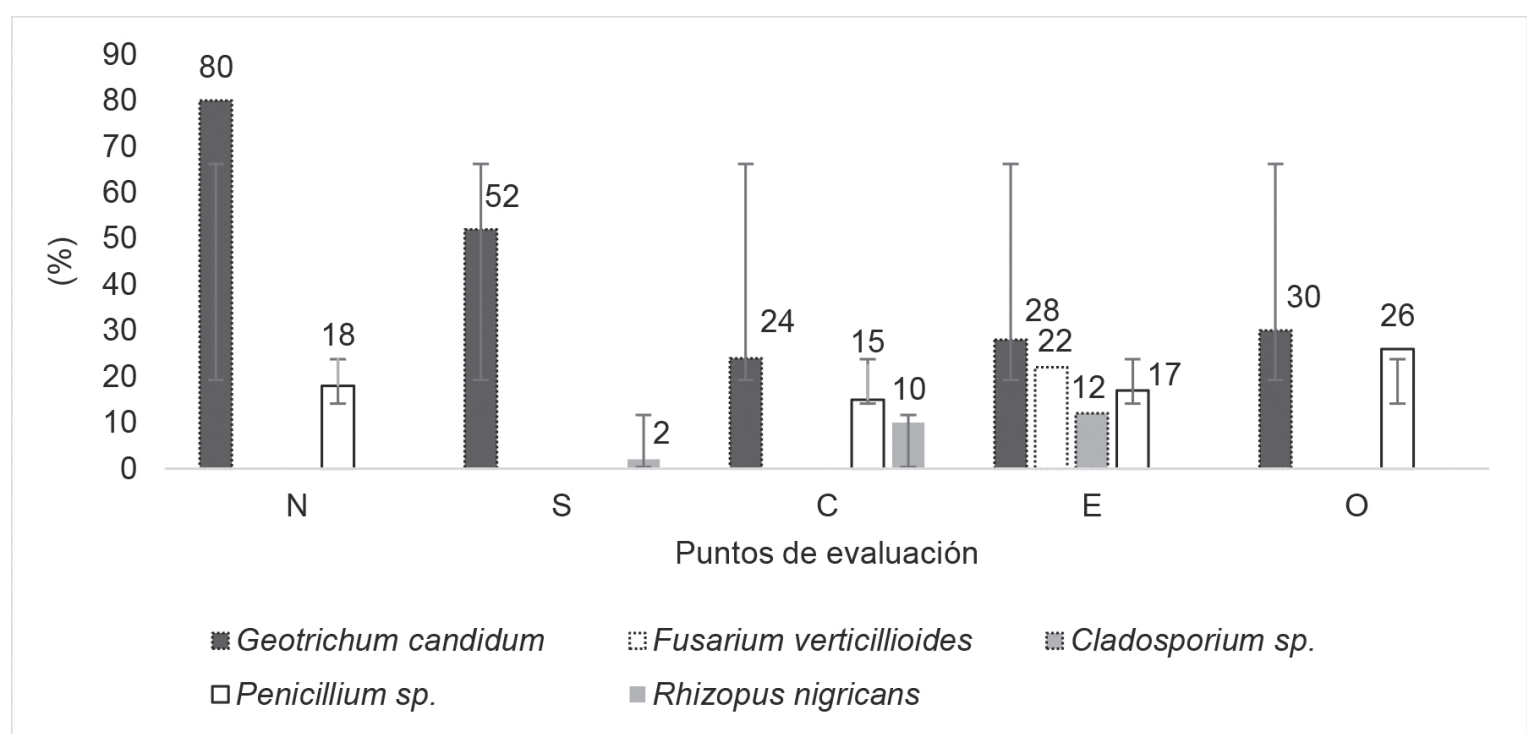

Figura 5. Porcentaje total de los hongos aislados en muestras de quesos artesanales en cada punto de evaluación ( $\mathrm{N}=$ Norte, $\mathrm{S}=\mathrm{Sur}, \mathrm{C}=$ Centro, $\mathrm{E}=$ Este y $\mathrm{O}=$ Oeste) . 


\section{Conclusión}

Los géneros y las especies identificadas podrían ser perjudiciales para la población que consume queso con mayor frecuencia, debido a que algunos son considerados como micotoxigénicos (Fusarium verticillioides y Penicillium sp.) y otros causantes de enfermedades en las personas (Geotrichum candidum y Rhizopus nigricans), por lo que es muy necesario implementar medidas de calidad que garanticen la producción de quesos inocuos para el consumo humano.

\section{Referencias bibliográficas}

[1] Mena J. El valor de los quesos artesanales en México (sitio de Internet). Disponible en: https:// www.forummexico.mx/gourmet/el-valor-delos-quesos-artesanales-en-m\%C3\%A9xico/. Acceso en 2019.

[2] Chacón L. Mexicanos consumen $6 \mathrm{~kg}$ de queso al año (sitio de Internet). Disponible en: https://manufactura.mx/industria/2014/11/07/ mexicanos-consumen-6-kg-de-queso-al-ano. Acceso 2019.

[3] Malcata FX, Kongo JM. Cheese: types of cheeses-soft. Encyclopedia of Food and Health. 2016

[4] OMS. Organización Mundial de la Salud. Enfermedades de transmisión alimentaria (sitio de Internet). Disponible en: https://www.who.int/ topics/foodborne_diseases/es/. Acceso en 2019.

[5] Ndoye B, Rasolofo E, LaPointe G, Roy D. A review of the molecular approaches to investigate the diversity and activity of cheese microbiota. Dairy Sci. Technol. 2011;91(5):495-24.

[6] Cécile L. Microflore du lait cru (sitio de Internet). Disponible en: http://iccheesemongers.com/ wp-content/uploads/Microfloredulaitcru-RMTjuillet2011BD.pdf. Acceso en 2019.

[7] Carrascal CA, Morales PA, Burbano RM. Manual de laboratorio: microbiología de alimentos. Bogotá, Colombia: Centro editorial javeriano CEJA; 2003.

[8] Binetti A, Carrasco M, Reinheimer J, Suárez V. Yeasts from autochthonal cheese starters: technological and functional properties. J. Appl. Microbiol. 2013;115(2):434-44.

[9] Padilla B, Manzanares P, Belloch C. Yeast species and genetic heterogeneity within Debaryomyces hansenii along the ripening process of traditional ewes' and goats' cheeses. Food Microbiol. 2014;38:160-166.
[10] Banjara N, Suhr MJ, Hallen-Adams HE. Diversity of yeast and mold species from a variety of cheese types. Curr. Microbiol. 2015;70:792-800.

[11]Ceugniez A, Drider D, Jacques P, Coucheney F. Yeast diversity in a traditional French cheese "Tomme d'orchies" reveals infrequent and frequent species with associated benefits. Food Microbiol. 2015;52:177-84.

[12] Dugat-Bony E, Garnier L, Denonfoux J, Ferreira S, Sarthou AS, Bonnarme P, Irlinger F. Highlighting the microbial diversity of 12 French cheese varieties. Int. J. Food Microbiol. 2016;238:265-73.

[13] Beresford T, Williams A. The microbiology of cheese ripening. Cheese: chemistry, physics and microbiology. 2004;1:287-318.

[14] Ross RP, Morgan S, Hill C. Preservation and fermentation: past, present and future. Int. J. Food Microbiol. 2002;79(1-2):3-16.

[15] Samson R, Hoekstra E, Frisvad JC. Introduction to food and airbone fungi. Séptima edición. Holanda: Editorial CBS; 2004.

[16] Barnett LH, Hunter BB. Illustrate genera of imperfect fungi. Minnesota, United States: The American Phytopathology Society Press; 1998.

[17] López MA. Diversidad de la microbiota fúngica del queso paipa fabricado en Pacho, Cundinamarca. rev. ion. 2011;24(1)77-84.

[18] Medina O, León-Montero $Y$, Delmonte $M$, Fernández P, Silva-A RA, Salcedo A. Mohos y levaduras en queso artesanal semiduro expendido en la ciudad de Maracaibo, estado Zulia, Venezuela. CIENCIA. 2014;22(4):197204.

[19] Beresford T, Fitzsimons N, Brennan N, Cogan T. Recent advances in cheese microbiology. Int. Dairy J. 2001;11:259-74.

[20] Arendrup MC, Boekhout T, Akova M, Meis JF, Cornely OA, Lortholary O. Sociedad Europea de Microbiología Clínica, Enfermedades Infecciosas Grupo de Estudio de Infecciones Fúngicas \& Confederación Europea de Micología Médica (2014). Directrices clínicas conjuntas de ESCMID y ECMM para el diagnóstico y el tratamiento de infecciones de levadura invasivas raras. 20 Suppl 3:76-98.

[21] Vasek MO, Cabrera RGJ, Coronel GJ, de Giori GS, Fusco AJV. Análisis de riesgos en la elaboración de queso artesanal de corrientes (Argentina). FACENA. 2004;20:3-22. 